\title{
RAYLEIGH WIND RETRIEVAL FOR THE ALADIN AIRBORNE DEMONSTRATOR OF THE AEOLUS MISSION USING SIMULATED RESPONSE CALIBRATION
}

\author{
Xiaochun Zhai ${ }^{1,2}$, Uwe Marksteiner ${ }^{1}$, Fabian Weiler ${ }^{1}$, Christian Lemmerz ${ }^{1}$, Oliver Lux ${ }^{1}$, Benjamin \\ Witschas $^{1}$, Oliver Reitebuch ${ }^{1 *}$ \\ ${ }^{I}$ Deutsches Zentrum für Luft- und Raumfahrt e.V. (DLR), Institut für Physik der Atmosphäre, \\ Oberpfaffenhofen 82234, Germany \\ ${ }^{2}$ Ocean University of China, College of Information Science and Engineering, Ocean Remote Sensing \\ Institute, Qingdao 266100, China \\ *Email: Oliver.Reitebuch@dlr.de
}

\begin{abstract}
Aeolus, launched on 22 August 2018, is the first ever satellite to directly observe wind information from space on a global scale. An airborne prototype called ALADIN Airborne Demonstrator (A2D) was developed at the German Aerospace Center (DLR) for validating the Aeolus measurement principle based on realistic atmospheric signals. However, atmospheric and instrumental variability currently limit the reliability and repeatability of the A2D instrument response calibration. In this study, a simulated Rayleigh response calibration (SRRC) is presented for resolving the limitations of A2D instrument response calibration.
\end{abstract}

\section{INTRODUCTION}

Continuous global wind measurement is of highest priority for improving the accuracy of numerical weather forecast as well as our knowledge of atmospheric dynamics [1]. Among the various techniques such as radiosonde, radar wind profiler and so forth, a spaceborne Doppler wind lidar is considered as the most promising one to meet the need of near-real time observations of global wind profiles. Aeolus, launched on 22 August 2018, is the first ever satellite to directly retrieve wind information from space on a global scale. The unique payload, the Atmospheric LAser Doppler INstrument (ALADIN), is a direct detection wind lidar operating at $355 \mathrm{~nm}$ which can probe the lowermost $30 \mathrm{~km}$ of the atmosphere from a 320 $\mathrm{km}$ orbit. It provides one component of the wind vector along the instrument's line-of-sight (LOS) with a vertical resolution of $0.25 \mathrm{~km}$ to $2 \mathrm{~km}$ and wind speed precision of $2 \mathrm{~m} / \mathrm{s}$ to $4 \mathrm{~m} / \mathrm{s}$ depending on altitude. The backscatter signals from particles
(Mie channel) and molecules (Rayleigh channel) are independently received by two different interferometers, that is, a Fizeau interferometer and double-edge Fabry-Perot interferometers (FPIs), respectively. The novel combination of these two techniques, which were not implemented in a wind lidar before, enlarges the observational altitude range compared to the coherent Doppler wind lidar which only relies on particulate scattering. In the frame of the Aeolus program, an airborne prototype of ALADIN called ALADIN Airborne Demonstrator (A2D) was developed at the German Aerospace Center (DLR) [2]. Due to its representative design and operating principle, the $\mathrm{A} 2 \mathrm{D}$ has provided valuable information on the wind measurement strategies of the satellite instrument as well as on the optimization of the wind retrieval and quality control algorithms $[3,4]$. The A2D was deployed in several ground and airborne campaigns over the last 12 years.

As opposed to coherent Doppler wind lidars where the Doppler frequency shift can be directly determined via the beat signal between the backscattered and emitted laser signal, the measured quantities from a direct detection wind lidar cannot be directly related to the Doppler frequency shift. The response calibration indicating the relationship between measured quantities and Doppler frequency shift is thus a prerequisite to retrieve wind information accurately. As for A2D, a small portion of transmitted laser radiation, referred to as internal reference signal, is collected and coupled into a multi-mode fiber firstly, and then entered the spectrometer optics [3], this optical path, called internal reference path, is used for internal reference laser frequency measurements. While 
atmospheric backscattered signal is firstly collected by telescope and then guided to the front and spectrometer optics in turn, the atmospheric path is used for the measurement of received atmospheric frequency. Therefore, the response calibrations for both internal reference and atmospheric path are prerequisite for accurate wind retrieval. In order to calibrate the ALADIN or A2D Rayleigh channel, different instrument calibration approaches have been carried out using both measurements and simulations [4,5]. In this paper, a simulated Rayleigh response calibration (SRRC) is presented to resolve the reliability and repeatability limitations of the A2D instrument response calibration caused by atmospheric and instrumental variability.

\section{METHODOLOGY}

Fig. 1 presents the measurement principle of the A2D Rayleigh channel. The emitted laser spectrum is depicted using a Gaussian function with a full width at half maximum (FWHM) of 50 $\mathrm{MHz}$, and the molecular Rayleigh backscatter spectrum is modeled using an analytical fit of the Tenti S6 line shape model [6-8] at $T=270 \mathrm{~K}$ and $P=700 \mathrm{hPa}$. In this case, it is assumed that there is no Mie contamination on the Rayleigh channel. The transmitted signals through each filter $\left(I_{A}\right.$, $I_{B}$ ), as indicated by light blue and magenta filled areas respectively, are proportional to the convolution of the respective filter transmission function and the line shape of the atmospheric backscatter signal. Therefore, the contrast between $I_{A}$ and $I_{B}$ represents a measure of the frequency shift between emitted laser pulse and molecular backscattered signal. Herein, the frequency-dependent Rayleigh response function is defined as:

$$
R(f)=\frac{I_{A}(f)-I_{B}(f)}{I_{A}(f)+I_{B}(f)}
$$

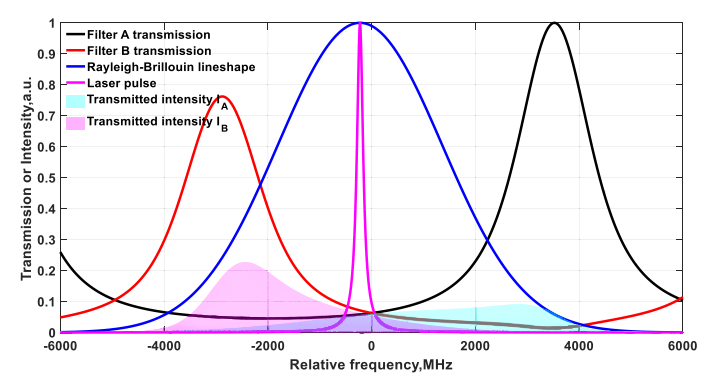

Fig. 1 Modeled spectral distribution of the transmitted laser pulse (pink line) and pure molecular backscatter (blue line) at $T=270 \mathrm{~K}, P=700 \mathrm{hPa}$. The Rayleigh channel transmission spectra of two FPIs are shown in black $T_{A}(f)$ and red $T_{B}(f)$ lines, respectively. The transmitted integrated intensities through FPI A and B are marked with light blue and magenta filled areas.

A frequency scan of the laser transmitter over a wide frequency range of $1.7 \mathrm{GHz}$ with step size of $25 \mathrm{MHz}$ is carried out to obtain the response function during $\mathrm{A} 2 \mathrm{D}$ instrument response calibration. During the instrument response calibration, the contribution of Doppler frequency shifts related to the motion of molecular or particle along the instruments' LOS direction needs to be eliminated. In practice, this is accomplished by flying a curve with a roll angle of $20^{\circ}$, resulting in approximately nadir pointing of the instrument and thus nearly zero wind contribution, provided that the vertical wind is negligible.

Following the procedure of the A2D instrument response calibration, SRRC can be carried out to obtain simulated response function based on Eq. (1). A linear least square fit is firstly applied to the simulated response function, and the sensitivity $\beta_{x}$ and intercept $\alpha_{x}$ are obtained from:

$$
\begin{gathered}
\beta_{x}=\frac{\partial R_{x}(f)}{\partial f}, x=A T M, I N T \\
\alpha_{x}=R_{x}(f=0),(3)
\end{gathered}
$$

where $x$ represents the simulated result derived from the internal reference or atmospheric path, respectively with $f$ being the relative frequency (wrt. frequency at the filter crosspoint). The nonlinearity $\gamma_{x}(f)$ is defined as the difference between $R(f)$ and the linear fit of simulated response function. Then a $5^{\text {th }}$ order polynomial fit is used to model $\gamma_{x}(f)$. The difference between $R(f)$ and the fitted simulated response function is defined as the response residual. The result shows a periodic fluctuation of the response residual for both the internal reference and the atmospheric path with quite small response value less than $1.5 \times 10^{-4}$.

As mentioned above, the realization of SRRC needs not only knowledge of the transmission 
characteristics of the FPIs, but also atmospheric temperature and pressure profiles for calculation of the molecular spectrum. These can be obtained from radiosondes, dropsondes or numerical weather prediction models. In the frame of the North Atlantic Waveguide and Downstream Experiment (NAWDEX) campaign in autumn of 2016, four aircraft equipped with diverse payloads were employed to investigate the influence of diabatic processes on the midlatitude weather [9]. The DLR Falcon 20 was deployed with the A2D and a well-established $2 \mu \mathrm{m}$ coherent Doppler wind lidar, offering an ideal platform to demonstrate the feasibility of $\mathrm{A} 2 \mathrm{D}$ in complex dynamic conditions. The other three aircraft were equipped with dropsonde dispensers to provide temperature, pressure, wind and humidity profiles, offering essential atmospheric temperature and pressure profiles for SRRC.

Since the transmission characteristics of the FPIs are different for the internal reference and atmospheric path due to slightly different illumination conditions of the different optical paths, different FPI transmission functions from different ground and airborne campaigns were used to calculate different simulated response functions. Each combination has been tested and analyzed. After comparison with the measured Rayleigh response function obtained during the NAWDEX campaign, it was found that the combination where the internal reference and atmospheric response functions were simulated using FPI parameters derived from the internal reference path during NAWDEX airborne campaign (INTA) and the atmospheric path during the BRAINS ground campaign (ATMG) [10] provides the best consistency with the measured one, which are used for initial SRRC determination. Fig. 2 shows the comparison of response functions between measurement and simulation, showing the best match for INTA and ATMG, respectively.
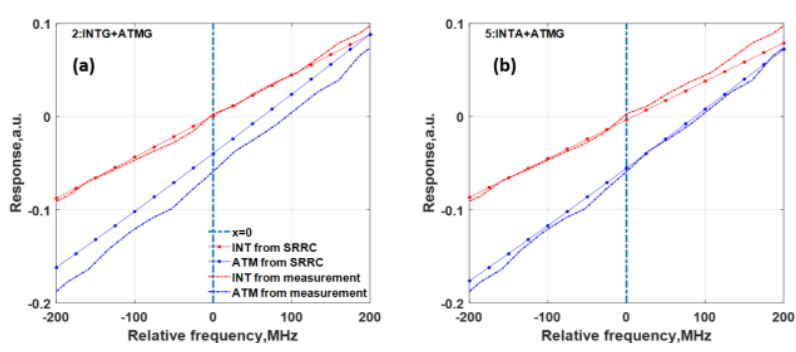

Fig. 2 The response functions of INT and $8^{\text {th }}$ range-bin ATM from calibration measurement (red dot and blue dot lines, respectively) and SRRC using different combinations of FPIs. (a) INTG+ATMG and (b)

INTA+ATMG for INT and ATM response simulation, respectively.

As slightly different illumination of the FPIs during Rayleigh response calibrations could yield large errors in retrieved LOS wind speed, an optimization of the FPI parameters is needed. Considering the optical path of the A2D Rayleigh channel, the FPI center frequency of the atmospheric path is sensitive to the incidence angle of the interferometer. Thus, the FPI transmission function for the atmospheric path could be adjusted for the center frequency of filter $\mathrm{A}$ or B. Assuming that the center frequencies of filters $\mathrm{A}$ and $\mathrm{B}$ have the same offset $\Delta f_{0}$ compared to the values obtained from ATMG and the parameters from INTA are regarded as ideal, Fig. 3 (a) (b) present the effect of $\Delta f_{0}$ on atmospheric response sensitivity and intercept, respectively. It can be seen the increase of center frequency of filter A and B results in a decrease of the response sensitivity and intercept. A cost function $F\left(\Delta f_{0}\right)$ is defined to determine optimized frequencies as follows:

$$
F\left(\Delta f_{0}\right)=\sum_{i=1}^{N}\left|V_{L O S, S R R C}(i)-V_{\text {LOS, dropsonde }}(i)\right|
$$

where $V_{L O S, S R R C}$ and $V_{\text {LOS,dropsonde }}$ are LOS wind speeds derived from SRRC and a dropsonde dataset used as a reference, while $i=1$ to $N$ denote the atmospheric range gates of the A2D. Fig. 3 (c) shows that $F\left(\Delta f_{0}\right)$ has its minimum when the center frequencies of both filters $\mathrm{A}$ and B increase by $20 \mathrm{MHz}$, corresponding to the optimization case for LOS velocity retrieval using SRRC.

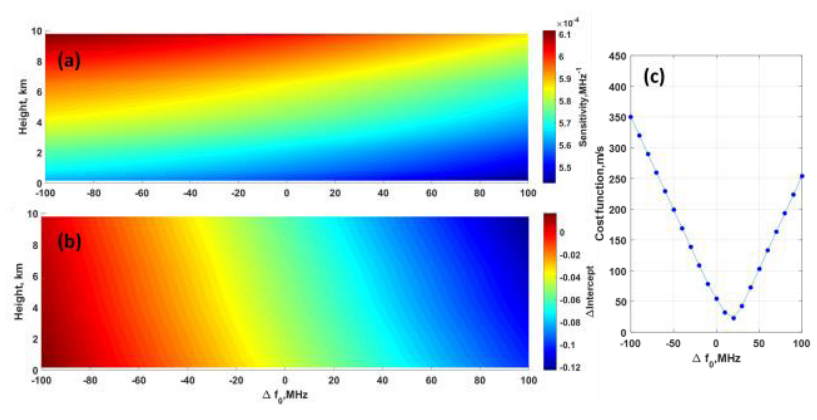


Fig. 3 The effect of the center frequency offset of filters A and B on the ATM response (a) sensitivity, (b) intercept, and (c) corresponding cost function.

\section{RESULTS}

In order to assess the accuracy of the LOS wind velocity retrieval from SRRC after FPIs optimization, Fig. 4 shows a case study comparison between measurement and simulation. Fig. 4 (a) (b) indicates that the sensitivity and intercept of the atmospheric path derived from SRRC have the same trend as the measured one, indicating the effect of atmospheric temperature and pressure on the response calibration. Because of the ground elevation limitation during A2D instrument response calibrations, the measured response below $2 \mathrm{~km}$ cannot be obtained, thus the response function at an altitude of $2 \mathrm{~km}$ is used for LOS wind retrieval below $2 \mathrm{~km}$, causing larger discrepancies compared to the dropsonde and coherent Doppler wind lidar dataset, as shown in Fig. 4 (c). As the SRRC is not affected by this limitation it improves the accuracy of A2D wind measurements especially at lower altitudes, as shown in Fig. 4 (c).

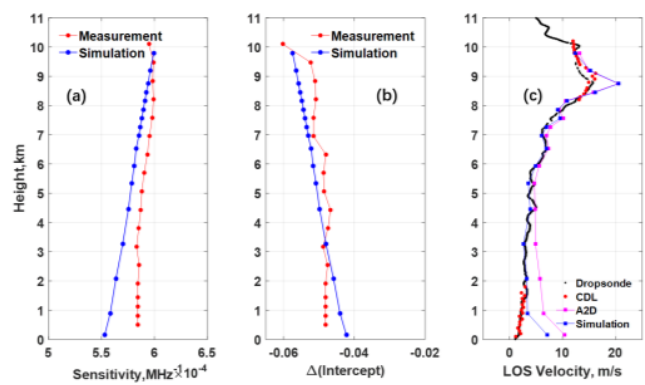

Fig. 4 Case study comparison of (a) sensitivity (b) intercept (c) LOS velocity between results from A2D Rayleigh channel measurement (red) and SRRC after FPIs optimization (blue). The LOS wind velocity from dropsonde (black) and coherent Doppler lidar (pink) are also presented.

\section{CONCLUSIONS}

The SRRC can resolve the reliability and repeatability limitations caused by atmospheric and instrumental variability and constraints during A2D instrument response calibrations. Unlike the A2D instrument response calibration, the SRRC is not affected by possible ground elevation limitations and can hence improve the accuracy of A2D wind measurements especially at lower altitudes. Further studies based on A2D SRRC will be performed with a focus on the atmospheric temperature and pressure effect, the Mie contamination correction and the optical properties of particles. From this, new sights into $\mathrm{A} 2 \mathrm{D}$ and Aeolus measurements and wind results are expected.

\section{ACKNOWLEDGEMENTS}

Xiaochun Zhai appreciates the support from the Chinese Scholarship Council (CSC) to conduct the research at DLR. DLR and ESA provided funding for the airborne campaigns mentioned in the text.

\section{REFERENCES}

[1] A. Stoffelen, et al. Bulletin of the American Meteorological Society, 86.1 (2005): 73-88.

[2] O. Reitebuch, et al. Journal of Atmospheric and Oceanic Technology, 26.12 (2009): 2501-2515

[3] O. Lux, et al. Atmospheric Measurement Techniques, 11.6 (2018): 3297-3322.

[4] U. Marksteiner, et al. Remote Sensing, 10.12 (2018): 2056.

[5] A. Dabas, et al. Tellus A: Dynamic Meteorology and Oceanography, 60.2 (2008): 206-215

[6] G. Tenti, et al. Canadian Journal of Physics, 52.4 (1974): 285-290.

[7] B. Witschas, et al. Applied Optics, 50.3 (2011): $267-270$

[8] B. Witschas, et al. Applied Optics, 50.29 (2011): 5758-5758.

[9] A. Schäfler, et al. Bulletin of the American Meteorological Society, 99.8 (2018): 1607-1637.

[10] B. Witschas, et al. Applied Optics, 51.25, (2012): 6207-6219. 